UC-700

Issued: September 1995

An Analysis of Containment, Surveillance, and Authentication for Software

William J. Hunteman 


\section{DISCLAIMER}

Portions of this document may be illegible in electronic image products. Images are produced from the best available original document. 


\section{CONTENTS}

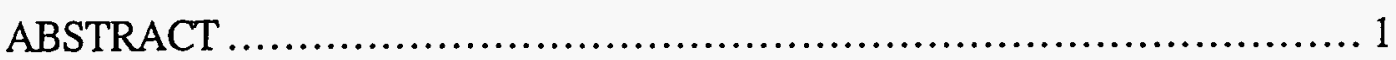

I. INTRODUCTION....................................................... 2

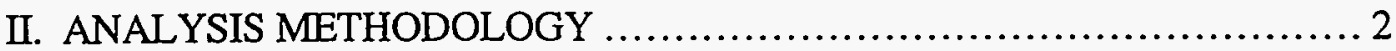

A. Assumptions and Threats .......................................... 2

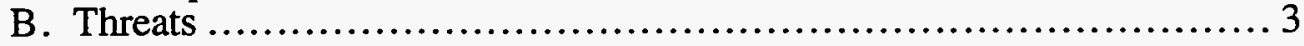

III. CONTAINMENT AND SURVEILLANCE FOR SOFTWARE .............. 3

A. Analysis ......................................................... 3

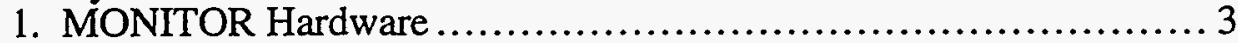

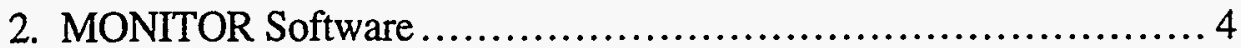

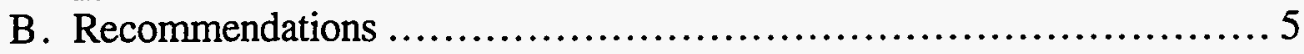

IV. SOFTWARE AUTHENTICATION .................................... 5

A. Analysis....................................................... 5

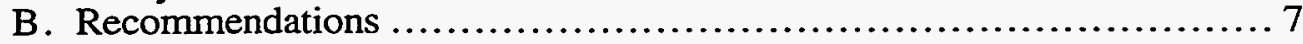

V. GENERAL CONCLUSIONS AND COMMENTS ...................... 7

A. Access Control .................................................... 7

B. Software Authentication............................................. 7

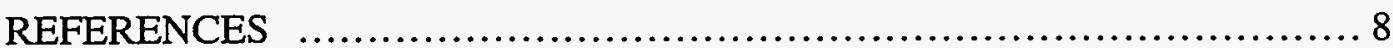




\title{
AN ANALYSIS OF CONTAINMENT, SURVEILLANCE, AND AUTHENTICATION FOR SOFTWARE
}

\author{
by
}

William J. Hunteman

\begin{abstract}
The "Protection, Containment, Surveillance, and Authentication (PCSA)" software was evaluated. The purpose of the evaluation was to determine the effectiveness of PCSA software in meeting the goals of a cost-effective, non-intrusive method for software authentication. Conclusions from the evaluation include

1. Commercially available software should be evaluated for the ability to provide the necessary control of access to and use of a PC.

2. The proposed software authentication methodology is not a cost-effective solution for the Agency and should be abandoned.
\end{abstract}




\section{INTRODUCTION}

This evaluation of the "Protection, Containment, Surveillance, and Authentication (PCSA)" software is based on the descriptions in [1] and [3]. The evaluation also includes the reviewer's general knowledge of computer security and information protection. The evaluation goal is to determine the effectiveness of the PCSA software in meeting the goals of a cost-effective, nonintrusive method for software authentication. The basic assumption in this evaluation is that the software to be authenticated is supplied by the facility operator and will be executed on facility provided systems. Section II describes the threats, requirements for safeguards software, and the overall evaluation approach. Section III addresses the MONITOR software used to control access to personal computers. Section IV addresses the proposed method of software authentication. Section V contains the conclusions and general recommendations.

\section{ANALYSIS METHODOLOGY}

The approach used to evaluate the PCSA system contains several steps. The first step is to define the threats that arise from the use of operator-provided hardware and software by the Agency. The second step is to evaluate PCSA components against those threats. Finally, several comments and recommendations are provided regarding the techniques for providing reasonable containment and surveillance of operator-provided software.

\section{A. Assumptions and Threats}

The stated goals for a PCSA system are as follows:

- Show that operator or state-authority hardware and software systems are reliable for use in the Agency's inspection program,

- Show that operator or state-authority hardware and software systems can be validated by the Agency,

- Use operator or state-authority hardware and software systems to reduce the Agency's overall costs, and

- Show that hardware and software systems developed and controlled by an operator or state authority can be authenticated for Agency use.

This analysis of the PCSA system includes the following objectives of a safeguards software protection system as defined in [2] .

- protect itself

- detect tampering with programs

- detect tampering with data

- assure inspector that all tampering has been detected

- permit authorized use of the PC

- log all events

- restrict log-file access to inspectors

- permit booting from an authorized operating system

- permit full access by an inspector

- permit each inspector to customize containment and surveillance

- protect new files created during operation

- be multi-layered

- restart following a system crash 
- restrict diskette activities to authorized operations

- prevent viral attack

- provide assurance that a PC is used only for authorized purposes

- detect unexpected actions from authorized software

- be fail safe

- record the passage of time

- detect attempts to access the PC by bypassing the operating system

- execute appropriate countermeasures

- permit mutual checking with safeguards programs

- be adaptable to different plants

- minimize inspector resources (time and equipment)

- minimize intrusion in normal operation of plant

- provide fast and accurate analysis of log file

- minimize overhead

\section{B . Threats}

The primary, and possibly only meaningful, threat is one of concealed diversion of nuclear material. Any PCSA system must meet the above objectives and effectively mitigate the concealed diversion threat.

A secondary threat that must be considered for any PCSA system is the possible unauthorized disclosure of operator or state authority proprietary information. The risk of unauthorized disclosure will increase significantly as more people are required to have access to or handle software design specifications and source statements.

\section{CONTAINMENT AND SURVEILLANCE FOR SOFTWARE}

The containment and surveillance method proposed in [1] requires the use of a MONTTOR program to control access to and use of operating system commands and application software.

\section{A. Analysis}

The MONITOR program is designed to control access to the personal computer (PC). The description of the MONITOR program supports the statement that it can control access to, and use of, the PC. However, the effectiveness of the MONITOR program depends on the use of the special hardware mentioned in [2] to prevent booting from an unauthorized operating system.

1. MONITOR Hardware. The requirement for special hardware will add costs to an operator's facility and will intrude on the facility operations. The special hardware is appropriate for Agency-owned PCs. If the PC is operator-owned, the special hardware will restrict the operator's flexibility in using the computing resources. For example, if the PC is not required for dedicated operations, 24 hours a day 7 days a week, the operator could use the PC for other activities. The special hardware will require that all activities, including those that are not safeguards related, be part of the PCSA program. The special hardware could also be viewed as an intrusion into the operator's computing resources. Operator concerns could include

- the potential for the special hardware to introduce changes to the software or data that could allow the diversion of nuclear material or embarrass the operator or State by distorting the data and 
- the potential for the special hardware to deliberately or accidentally modify the software or data and thereby disrupt the operation of the facility.

The operator can be expected to require assurances from the Agency that the special hardware is unlikely to affect the operation of the facility. The need for the assurances would be repeated each time the operator upgraded the hardware or software in PCs where the special hardware was installed. It is reasonable to expect that the special hardware will have limited functionality and be relatively easy to understand. However, the development of the assurances will require the Agency, or an organization paid by the Agency, to frequently evaluate the hardware with the latest version of PC hardware and software. For example, if the hardware was used in a PC with Microsoft Windows, every time the Windows software was upgraded the hardware must be tested to ensure that it is compatible with the latest version. Also, unless the Agency is able to restrict the PC configuration, including brands, then the hardware must be tested with most of the PCs available in the world market. Some of the testing requirements may be predictable based on the Agency's early involvement in the design of new or modifications to facilities under safeguards.

The normal maintenance of PCs will also require the Agency to test the hardware against most of the commercially available PCs. For example, if a PC containing the special hardware fails in a facility, the operator may decide to simply replace the unit with another one. In this case, configuration controls will be necessary to ensure that the special hardware is properly installed in the new PC. The new PC may also be significantly different from the previous unit. If the original PC used an INTEL 386 processor, the operator may be unable or unwilling to replace the unit with a PC containing the 386 . PCs containing 486 or 586 processors may be the only available units and the Agency would be required to assure the operator that the special hardware would operate correctly with the new unit.

2. MONITOR Software. The MONITOR program appears to be designed to supply the features and assurances necessary to provide a role-based protection environment. These features and assurances include the following, from the objectives of a safeguards software protection system [2].

- protect itself

- permit authorized use of the PC

- $\log$ all events

- restrict $\log$ file access to inspectors

- permit full access by an inspector

- protect new files created during operation

- be multi-layered

- restart following a system crash

- restrict diskette activities to authorized operations

- prevent viral attack

- provide assurance that a PC is used only for authorized purposes

- detect unexpected actions from authorized software

- be fail safe

- record the passage of time

- detect attempts to access the PC by bypassing the operating system

- execute appropriate countermeasures

- permit mutual checking with safeguards programs

- be adaptable to different plants

- minimize inspector resources (time and equipment) 
- minimize intrusion in normal operation of plant

- minimize overhead

Additional detailed analysis and testing by an independent objective organization is necessary before the claims for MONITOR can be accepted. This evaluation would be designed to demonstrate, using a variety of analysis techniques, that the MONITOR program correctly implements the design objectives and that the software cannot be bypassed.

The MONITOR program is designed to work in an MS-DOS environment. However, advances in computer hardware and software technology are moving most PC activities to a Microsoft Windows environment. These advances will require modification of MONITOR. The United States National Computer Security Center (NCSC) is nearing the completion of evaluation of the security functions of Microsoft Windows NT. Informal discussion with NCSC personnel indicates that Windows NT is expected to receive a $\mathrm{C} 2$ rating. This rating is assigned if the system provides discretionary access control between users and files, identification and authentication of users, auditing of user activities, and protection of audit data from unauthorized access. The network functions of Windows NT are also being tested and are expected to receive a rating of C2. The additional features provided in Windows NT, such as virus detection and restrictions on user activities, duplicate many of the functions of the MONITOR software.

\section{B . Recommendations}

The requirements should be analyzed to compare the MONITOR functions to the capabilities of commercially available software. The results of this analysis should be used to determine if the MONITOR program should be upgraded or abandoned.

If the MONTTOR program is upgraded to the Windows environment, the new software should be evaluated in detail to establish that the features and assurances function as described and cannot be easily defeated. This evaluation must be based on design specifications and analysis of the source code. This evaluation must be conducted by an independent organization.

If the MONITOR program will be used in DOS environments, the software should be evaluated in detail to establish that the features and assurances function as described and cannot be easily defeated. This evaluation must be based on design specifications and analysis of the source code. This evaluation must be conducted by an independent organization.

\section{SOFTWARE AUTHENTICATION}

The Software Authentication (SA) methodology requires the use of an Agency-supplied routine that is inserted into the operator or state-authority software to establish assurances that the output of a program was based on the inputs to that program.

\section{A. Analysis}

The operator or state-authority software is modified to call the Agency-supplied routine whenever a file is accessed by the program. The Agency routine computes an authentication number for the file, which is returned to the program. The program repeats this process for all files and then calls the routine to produce the combined authentication number (CAN), which is derived from the authentication numbers for each of the files and the safeguards program. The INSPECT program is used to examine the CAN for evidence of tampering with the safeguards program or data files. 
SA appears to be designed to supply the features and assurances necessary to detect tampering with programs and data files. These features and assurances include the following from the objectives of a safeguards software protection system [2].

- protect itself

- detect tampering with programs

- detect tampering with data

- assure the inspector that all tampering has been detected

- permit each inspector to customize C/S

- protect new files created during operation

- be multi-layered

- restart following a system crash

- be adaptable to different plants

- minimize inspector resources (time and equipment)

- minimize overhead

$\mathrm{SA}$, as described in [3], meets the above requirements. However, it does not meet the requirement to minimize the intrusion into normal operation of the plant and will have substantial impact on Agency resources.

The modification of the safeguards program by inserting calls to the Agency routine will require that all source statements in the software be examined by Agency personnel. The examination is necessary to ensure that the calls have been correctly inserted into all locations dealing with data files. The examination is also required to show that the safeguards program performs the correct computations and does not bypass any of the data-file authentication operations. The Agency must either dedicate the people to learn the details of the safeguard program or trust the operator or state-authority personnel to correctly call and use the Agency routine. If the Agency expects to depend upon the results of the safeguards program, this examination will be necessary regardless of which authentication method, if any, is used. Several studies conducted by the computer security community have demonstrated the difficulty of searching source statements for unauthorized operations. Once the software has been examined, it must be tightly controlled to prevent unauthorized access or changes until it has been compiled or assembled.

Once the safeguards program has been correctly modified, the safeguard program must be compiled or assembled. If the source statements are returned to the operator or state-authority, all control will be lost and there is no assurance that the source statements examined by the Agency are the same ones compiled by the operator. The loss of control would permit other unknown software modifications or the use of compilers and assemblers that could also alter the safeguards program. Several studies and experiments by the computer security community have demonstrated the ability to make undetected changes to programs by modifying compilers to insert computer instructions that did not exist in the source statements of either the compiler or the program being compiled. The only methods to ensure that the safeguards program source statements are compiled correctly are (1) compile or assemble the safeguards program at the Agency and return only the executable file to the operator, or (2) require that the operator's compiler or assembler utilize the software authentication methodology. Option one will require extensive Agency resources to provide the necessary hardware and software to compile each safeguards program used at every plant under Agency safeguards. Option two would require that the supplier of the compiler or assembler become involved with the Agency. Economic competition would either force the supplier to increase their charges to the plant or force the supplier to not sell their products to the plant operator.

The software reviews and compilation issues must addressed every time the software or its operating environment is changed. For example, every time the software is corrected, the software 
must be reviewed and compiled under controlled conditions to ensure that no unauthorized modifications are made. Any change in the operating system or software libraries used by the safeguards program would require a re-examination of the source statements and possibly a new compilation.

\section{B. Recommendations}

The proposed software authentication methodology will result in substantial increases in Agency personnel and computer resources or will require the Agency to accept assertions from the plant operators or state authorities that the safeguards software has been properly modified to provide the requested authentication. Another less-intrusive, more cost-effective approach to software authentication should be developed.

\section{GENERAL CONCLUSIONS AND COMMENTS}

\section{A. Access Control}

Commercially available software should be evaluated for the ability to provide the necessary control of access to and use of a PC. This activity should include a survey of the commercially available software followed by an evaluation of functionality in the identified software components.

If commercially available software will provide the necessary access controls, the software should be tested by the Agency if the software has not been evaluated by another organization.

If commercial software is not available, the MONITOR software should be redesigned and implemented to support the Microsoft Windows environment. Once the development is completed, the software must be tested by an independent organization.

\section{B . Software Authentication}

The proposed software authentication methodology is not a cost-effective solution for the Agency and should be abandoned. An alternative, less-intrusive, more-cost-effective approach must be developed if the Agency expects to use operator or state authority supplied systems. Other possible methods include

- use of file system integrity checks, such as the Tripwire tool developed at Purdue University, which monitors a designated set of files and directories for changes.

- use of digital signatures for the safeguards program, and

- use of encryption techniques to deter unauthorized changes to the safeguards program.

Each of these methods, including the proposed authentication method, will require the Agency to establish that the operator's program will produce the output desired by the Agency. Any software authentication method should concentrate on minimizing the Agency costs after the examination is completed. 


\section{REFERENCES}

[1] J. C. Andress, "Containment and Surveillance for Software," AEA FS 0193(WF), SRDPR207. AEA-RS 1306 (July 1993).

[2] J. C. Andress, "Safeguards Software C/S Checklist," Control and Dynamics Department Note 274 (February 1993).

[3] J. C. Andress, "Computer Software Authentication," AEA FS (106)WF, SRDP-R186, AEA-RS-6043 (March 1992). 